ECCOMAS

Proceedia
UNCECOMP 2021

$4^{\text {th }}$ ECCOMAS Thematic Conference on Uncertainty Quantification in Computational Sciences and Engineering M. Papadrakakis, V. Papadopoulos, G. Stefanou (eds.) Streamed from Athens, Greece, 28 -30 June 2021

\title{
INVERSE PROBLEMS FOR STOCHASTIC NEUTRONICS
}

\author{
Corentin Houpert $^{1}$, Josselin Garnier ${ }^{2}$, Philippe Humbert ${ }^{3}$ \\ ${ }^{1} \mathrm{CEA}, \mathrm{DAM}, \mathrm{DIF}$ and IP Paris, École polytechnique \\ F-91297 Arpajon \\ e-mail: corentin.houpert@cea.fr \\ ${ }^{2}$ IP paris, École polytechnique \\ Route de Saclay, 91120 Palaiseau \\ e-mail: josselin.garnier@polytechnique.edu \\ ${ }^{3}$ CEA, DAM, DIF \\ F-91297 Arpajon \\ e-mail: philippe.humbert@cea.fr
}

\begin{abstract}
Fissile matter detection and characterisation are crucial issues; especially in nuclear safety, safeguards, matter comptability, reactivity measurements. In this context, we want to identify a source of fissile matter knowing external measures such as instants of detection of neutrons during an interval of measure. Thus we observe the neutrons detection times emitted by the fissile matter and going through the detector, then we compute the moments of the empirical distribution of the number of neutrons detected during a time gate T. In order to identify the source we have to get the following parameters: the multiplication factor $k$ of the system, the intensity of the source $S$, the fission efficiency $\varepsilon_{F}$.

Given the parameters of the source there are some models that allow us to predict the moments of counted number of neutrons during a time gate T. We consider a point model stating monokinetic neutrons are moving in an infinite, isotropic and homogeneous medium. The method makes it possible to compute the first moments of the count number distribution.

Then, given the moments of counted number of neutrons during a time gate $T$ we want to get the parameters of the fissile source. In order to achieve this goal, we will use the following method

- Bayesian approach in order the get the distribution of parameters. The a posteriori distribution is non-trivial, samples can be achieved with Markov Chain Monte-Carlo methods with covariance matrix adaptation (MCMC with CMA).
\end{abstract}

Keywords: Uncertainty Quantification, Bayesian inverse problems, Adaptative Metropolis, Covariance matrix adaptation, Stochastic neutronics, Neutron point model approximation 


\section{Nomenclature}

\section{Nuclear constants}

$\alpha \quad$ Decreasing coefficient of the neutronic system

$\bar{\nu} \quad$ Mean number of neutrons emitted by a fission event

$\bar{\nu}_{S} \quad$ Mean number of neutrons emitted by a source event

$\lambda_{C} \quad$ Capture rate by time unit

$\lambda_{F} \quad$ Fission rate by time unit

$D_{2 S} \quad$ Diven factor of the source of order 2

$D_{2} \quad$ Diven factor of the fission of order 2

$D_{3 S} \quad$ Diven factor of the source of order 3

$D_{3} \quad$ Diven factor of the fission of order 3

$f_{\nu} \quad$ Probability the fission emits $\nu$ neutrons

$f_{\nu, S} \quad$ Probability that source emits $\nu$ neutrons during a source event

$p \quad$ Probability that a neutron causes a fission

\section{Nuclear parameters}

p Vector of the parameters of the system

p* Vector of the parameters of the system to estimate

$\varepsilon_{C} \quad$ Capture efficiency

$k \quad$ Multiplication factor

$S \quad$ Intensity of the source (neutron/units of time)

\section{Observations and model outputs}

M Vector of the first three simple statistical moments, the model

$\hat{\mathbf{M}} \quad$ Vector of the first three simple empirical moments, the measures

$T \quad$ Time gate (units of time)

\section{Introduction}

We are interested in fissile matter detection and characterisation. We want to determine the fissile source with external measures. Times of neutron detections during an interval of measure provides the observations.

We study here an inverse problems under limited data. The inverse problem is ill-posed, getting the entries of the model is challenging. To tackle this issue we use bayesian methods, the a posteriori distribution provides the relative probability of the entries knowing the measures, our observation [12]. In order to sample this distribution we will use a MCMC method: the Metropolis-Hastings algorithm.

Since the distribution is degenerate when the measures are extensive, we will use an AdaptiveMetropolis algorithm with Covariance Matrix Adaptation. 
The paper is organized as follows. First, we introduce the neutron point model and expose the expressions of the simple moments of the neutrons count distribution [7].

Secondly, we will recall Bayes rules, present the requirements for the sampling and expose the given covariance for the measures. And we will also present the sampling of the a posteriori distribution, the discretisation with 3 parameters.

Finally, we will expose the results of the sampling with a benchmark. We will analyse the features of the sampled distribution with an explicit sampling and MCMC one, and settle how the work can be improved.

\section{Stochastic neutronics problem, forward problem}

The simplest model in neutronics is the point model approximation.

Definition 2.1 Point model [11]

The medium is infinite, homogeneous and isotropic. The neutrons are supposed point particles moving at the same speed. Moreover, we consider the neutron's life ends with a capture (with or without a detection) or a fission. These events are poissonian type. Neutrons are produced by fission and by the Poisson or compound Poisson type sources. A fission chain is modeled as a branching process.

The model is governed by the following parameters

\subsection{Source}

We model the source as a compound Poisson process with a strength

\section{Definition 2.2}

$$
S:=\text { Intensity of the compound Poisson process }
$$

The probability distribution of the number of neutrons emitted by a source event is given by

$$
f_{\nu, S}
$$

where $\nu$ goes from 0 to the maximum number of neutrons emitted by the source $\nu_{\max , S}$. The mean number of neutrons emitted by one source event is

$$
\bar{\nu}_{S}:=\sum_{\nu=0}^{\nu_{\max , S}} \nu f_{\nu, S} .
$$

From this, we can derive the following nuclear constants.

Definition 2.3 The Diven factors of order 2 and 3 of the source probability distribution are

$$
D_{2 S}:=\frac{\sum_{\nu} \nu(\nu-1) f_{\nu, S}}{\bar{\nu}_{S}^{2}}, D_{3 S}:=\frac{\sum_{\nu} \nu(\nu-1)(\nu-2) f_{\nu, S}}{\bar{\nu}_{S}^{3}}
$$




\subsection{Fission}

Definition 2.4 Let $p$ be the probability that a neutron causes a fission (so $1-p$ is be the probability that a neutron be captured).

The probability distribution of the number of neutrons produced by a fission is

$$
f_{\nu}
$$

where $\nu$ goes from 0 to the maximum number of neutrons emitted by the fission $\nu_{\max }$, and

$$
\bar{\nu}:=\sum_{\nu=0}^{\nu_{\max }} \nu f_{\nu}
$$

the mean number of neutrons emitted by one source event. When a fission occurs $\bar{\nu}$ neutrons are emitted on average.

Then

$$
k:=\bar{\nu} p
$$

is the mean number of children of a neutron. We will call it the multiplication factor [2].

In our case $0<k<1$, so the system is stationary which is the most important configurations for nuclear safety applications [9].

As previously, we obtain the formulas of the Diven factors of the fission of order 2 and 3.

$$
D_{2}:=\frac{\sum_{\nu} \nu(\nu-1) f_{\nu}}{\bar{\nu}^{2}}, D_{3}:=\frac{\sum_{\nu} \nu(\nu-1)(\nu-2) f_{\nu}}{\bar{\nu}^{3}}
$$

Definition 2.5 The fission rate is

$$
\lambda_{F}
$$

\subsection{Capture}

The neutron count is the action of detecting the neutron presence.

Definition 2.6 The capture rate is

$$
\lambda_{C}
$$

Definition 2.7 We define the capture efficiency by

$$
\varepsilon_{C}:=\text { Probability that a captured neutron is detected }
$$

This efficiency is linked to the fission one $\varepsilon_{F}$ by the equality

\section{Definition 2.8}

$$
\varepsilon_{F}:=\text { Detector efficiency }=\frac{\lambda_{C} \varepsilon_{C}}{\lambda_{F}}
$$

this is the ratio of the mean number of detections over the mean number of induiced fissions. 


\subsection{Measurements}

We get our observations from a detector with Helium 3 [15]. Neutronicians choose this element because its cross-section is large, so the capture probability is high. Neutrons are absorbed in the detector with the reaction

$$
{ }_{2}^{3} \mathrm{He}+{ }_{1}^{0} \mathrm{n} \rightarrow{ }_{1}^{1} \mathrm{H}+{ }_{1}^{3} \mathrm{H}+765 \mathrm{keV}
$$

then the proton emerging from the reaction causes an electric current. During a time interval of duration $T_{\text {meas }}$, each instant of detection is stored as a list in file (see fig. 1). Then we obtain $n=\left\lfloor\frac{T_{\text {meas }}}{T}\right\rfloor$ realizations of $N_{[0, T]}$ the counted neutrons during a time gate $T$, and we compute the empirical moments of this distribution

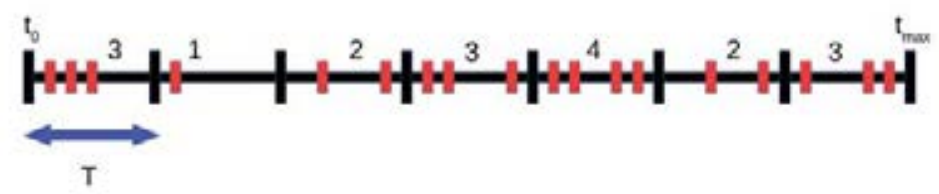

Figure 1: A measurement during $t_{0}$ and $t_{\max }=t_{0}+T_{\text {meas }}$, with a time gate $T$

\subsection{Forward model}

Definition 2.9 Let $N_{[0, T]}$ be the random variable representing the neutron counts during $T$. The three first associated moments of this distribution are

- $\mathbb{E}\left[N_{[0, T]}\right]:=$ the first moment of the neutron counted during $T$ distribution

- $\mathbb{E}\left[N_{[0, T]}^{2}\right]:=$ the second simple moment of the neutron counted during $T$ distribution

- $\mathbb{E}\left[N_{[0, T]}^{3}\right]:=$ the third simple moment of the neutron counted during $T$ distribution

These are the outputs of our model.

In the case of the point model the simple moments can be expressed as a function of the socalled Feynman moments $Y_{2}(T), Y_{3}(T)$ [9].

Proposition 2.10 The first three simple moments of $N_{[0, T]}$ are of the form

$$
\begin{aligned}
& \mathbb{E}\left[N_{[0, T]}\right]=\bar{\nu}_{S} S \frac{\varepsilon_{F} k}{(1-k) \bar{\nu}} T \\
& \mathbb{E}\left[N_{[0, T]}^{2}\right]=\mathbb{E}\left[N_{[0, T]}\right]\left(1+\mathbb{E}\left[N_{[0, T]}\right]+Y_{2}\right) \\
& \mathbb{E}\left[N_{[0, T]}^{3}\right]=\mathbb{E}\left[N_{[0, T]}\right]\left(1+3 Y_{2}+Y_{3}\right)+3 \mathbb{E}\left[N_{[0, T]}\right]^{2}\left(1+Y_{2}\right)+\mathbb{E}\left[N_{[0, T]}\right]^{3}
\end{aligned}
$$

where the Feynman moments are given by

$$
\begin{aligned}
Y_{2}(T) & =\frac{\varepsilon_{F} D_{2} k}{(k-1)^{2}}\left(1-\rho \frac{\bar{\nu}_{S} D_{2 S}}{\bar{\nu} D_{2}}\right)\left(1-\frac{1-e^{-\alpha_{Y} T}}{\alpha_{Y} T}\right) \\
Y_{3}(T) & =3\left(\frac{\varepsilon_{F} D_{2} k}{-(1-k)^{2}}\right)^{2}\left(1-\rho \frac{\bar{\nu}_{S} D_{2 S}}{\bar{\nu} D_{2}}\right)\left(1+e^{-\alpha_{Y} T}-2 \frac{1-e^{-\alpha_{Y} T}}{\alpha_{Y} T}\right) \\
& -\frac{\varepsilon_{F} D_{3} k^{3}}{(k-1)^{3}}\left(1-\frac{k-1}{k} \frac{\bar{\nu}_{S}^{2} D_{3 S}}{\bar{\nu}^{2} D_{3}}\right)\left(1-\frac{3-4 e^{-\alpha_{Y} T}+2 e^{-2 \alpha_{Y} T}}{\alpha_{Y} T}\right)
\end{aligned}
$$

where $\alpha_{Y}=\lambda_{C}+\lambda_{F}(1-\bar{\nu})$ 
A proof of this result can be found in [7].

Our forward model is

$$
\begin{aligned}
\mathbf{M}: \mathbb{R}^{3} & \rightarrow \mathbb{R}^{3} \\
\mathbf{p} & \mapsto \mathrm{M}(\mathbf{p})
\end{aligned}
$$

where $\mathbf{p}=\left(\varepsilon_{C}, k, S\right)$ and $\mathbf{M}_{j}(\mathbf{p})=\mathbb{E}\left[N_{[0, T]}^{j}\right]$.

\section{Bayesian inverse problem}

\subsection{Bayes principle}

We have the observations $\hat{\mathbf{M}}$ which are the estimated moments of $N_{[0, T]}$. Bayes theorem [13] states

$$
\underset{\text { a posteriori distribution }}{\mathbb{P}(\mathbf{p} \mid \hat{\mathbf{M}})} \propto \underset{\text { likelihood a priori distribution }}{\mathbb{P}(\hat{\mathbf{M}} \mid \mathbf{p}) \quad \mathbb{P}(\mathbf{p})}
$$

where the likelihood and the a priori distribution are as follows

1. Thanks to the Central Limit Theorem, given the parameter $\mathbf{p}$ the measures are Gaussian with mean $\mathbf{M}(\mathbf{p})$ and covariance $\frac{1}{n} \operatorname{Cov}(\mathbf{p})$ where $\mathbf{M}$ refers to the expression of the exact simple moments of the distribution of $N_{[0, T]}, \operatorname{Cov}(\mathbf{p})$ the covariance matrix of the three first simple moments, $n$ the number of realizations.

This gives explicitly

$$
\mathbb{P}(\hat{\mathbf{M}} \mid \mathbf{p}) \propto \frac{1}{\sqrt{\operatorname{det}\left(\frac{1}{n} \mathbf{C o v}(\mathbf{p})\right)}} e^{-\frac{1}{2}{ }^{t}(\hat{\mathbf{M}}-\mathbf{M}(\mathbf{p})) \operatorname{Cov}(\mathbf{p})^{-1}(\hat{\mathbf{M}}-\mathbf{M}(\mathbf{p})) n}
$$

which is the expression of the likelihood up to a multiplicative constant.

The computation of $\operatorname{Cov}(\mathbf{p})$ needs the expression of the simple moments up to the order 6 , and this is too complex to be computed analytically. So we will use the empirical covariance matrix $\widehat{\operatorname{Cov}}$.

$$
\tilde{\mathbb{P}}(\hat{\mathbf{M}} \mid \mathbf{p}) \propto \frac{1}{\sqrt{\operatorname{det}\left(\frac{1}{n} \widehat{\mathbf{C o v}}\right)}} e^{-\frac{1}{2}^{t}(\hat{\mathbf{M}}-\mathbf{M}(\mathbf{p})) \widehat{\operatorname{Cov}}^{-1}(\hat{\mathbf{M}}-\mathbf{M}(\mathbf{p})) n}
$$

2. The a priori distribution is assumed to be uniform on $\left[\varepsilon_{C, \min }, \varepsilon_{C, \max }\right] \times\left[k_{\min }, k_{\max }\right] \times$ $\left[S_{\min }, S_{\max }\right]$.

Our goal is to sample the a posteriori distribution 16. We will use two different methods: a discrete sampling with a regular mesh and Adaptive Metropolis with Covariance Matrix Adaptation.

\subsection{Explicit sampling of the a posteriori distribution}

A simple way to obtain the explicit sampling of the a posteriori distribution is to use a regular mesh of the domain $\left[\varepsilon_{C, \min }, \varepsilon_{C, \max }\right] \times\left[k_{\min }, k_{\max }\right] \times\left[S_{\min }, S_{\max }\right]$ and compute the a posteriori distribution on each point of the mesh. The computations were done with $N_{e}$ points in each directions. The overall number of evaluations of the forward model 15 is therefore $N_{e}^{3}$. We also compute the moments in order to have some quantitative information: mean, variance, expectation to be compared with MCMC results.

Remark 3.1 By 16 the computation of the likelihood is true up to a multiplicative constant. 


\subsection{MCMC sampling of the a posteriori distribution}

The principle of the method is to build a Markov chain that has the target distribution as its stationary distribution. Hence one can obtain a sample of the target distribution by sampling and recording states from the chain. Various algorithms exist for constructing such Markov chains, including the Metropolis-Hastings $(\mathrm{MH})$ algorithm. The states of the $\mathrm{MH}$ chain are produced iteratively. At each iteration, the algorithm picks a random proposal according to some instrumental distribution that may depend on the current sample value. The proposal is the candidate for the next sample value and it is either accepted (in which case the proposal value is used in the next iteration) or rejected (in which case the proposal value is discarded, and the current value is used in the next iteration) with some probability. The probability of acceptance is determined by comparing the values of the target density at the current and proposal values so as to ensure that the $\mathrm{MH}$ chain has the target distribution as its stationary distribution.

We implement here a specific MCMC method [10]: the Adaptive Metropolis algorithm with Covariance Matrix Adaptation in order to sample a target distribution $\pi$.

The adaptation uses the Covariance matrix of the all the points proposed by the instrumental law and accepted by the rejection procedure in order to accept more.

We implemented the following algorithm using [1] and [6]. Here

$$
\mathbf{p}=\left(p_{1}, p_{2}, p_{3}\right)
$$

and we define $p_{1, \min }=\varepsilon_{C, \min }, p_{1, \max }=\varepsilon_{C, \max }, p_{2, \min }=k_{\min }, p_{2, \max }=k_{\max }, p_{3, \min }=$ $S_{\min }, p_{3, \max }=S_{\max }$ The target distribution is denoted $\pi$.

We first initiate

- The empirical acceptance $x_{\text {rate }}$.

- The initial scale factor of the instrumental law frac

- The target acceptance rate $x_{o b j}$

- The frequency of update of the scale factor $N_{M C, 1}$

- The burnin phase duration $N_{b p}$

- The initial parameter $\mathbf{p}_{0}$ is chosen with the uniform distribution over $\bigotimes_{k=1}^{3}\left[p_{k, \min }, p_{k, \max }\right]$

Iteration $i \rightarrow i+1$

1. During $i \leq N_{b p}$, we use as instrumental law

$$
\mathbf{q}_{i+1} \sim \mathcal{N}\left(\mathbf{p}_{i}, \operatorname{frac}^{2} \mathbf{C}_{b i}\right)
$$

where $\mathbf{q}_{i+1}$ the proposal, $\mathbf{p}_{i}$ the last accepted point, $\mathbf{C}_{b i}=\operatorname{diag}\left(\left(\left(p_{k, \max }-p_{k, \min }\right)^{2}\right)_{k=1}^{3}\right)$.

2. After the burnin we use the instrumental law as in algorithm 4 of [1]

$$
\mathbf{q}_{i+1} \sim \mathcal{N}\left(\mathbf{p}_{i}, \operatorname{frac}^{2} \mathbf{C}_{i}\right)
$$

where $\mathbf{C}_{i}$ is defined by 24 . 
3. Finally we compute the acceptance rate $\alpha$ using the likelihood ratio of the proposal and the previous accepted point

$$
\alpha\left(\mathbf{q}_{i+1}, \mathbf{p}_{i}\right)=\min \left(1, \frac{\pi\left(\mathbf{q}_{i+1}\right)}{\pi\left(\mathbf{p}_{i}\right)}\right)
$$

4. The acceptance-rejection criterion

$$
\begin{aligned}
& u \sim \mathcal{U}([0,1]) \\
& \text { If } u \leq \alpha\left(\mathbf{q}_{i+1}, \mathbf{p}_{i}\right) \text { then } \mathbf{q}_{i+1} \text { is accepted: } \mathbf{p}_{i+1}=\mathbf{q}_{i+1} \text { otherwise } \mathbf{p}_{i+1}=\mathbf{p}_{i}
\end{aligned}
$$
is applied

5. We update the scale factor when $i \equiv 0\left(\bmod N_{M C, 1}\right)$. We update the scale factor frac

$$
\begin{aligned}
& f r a c=f r a c \exp \left(x_{\text {rate }}-x_{o b j}\right) \\
& x_{\text {rate }}=\frac{\text { Number of acceptation }}{\text { Number of iterations }}
\end{aligned}
$$

This is an algorithm with global scaling and with vanishing adaptation so the ergodicity of the algorithm is achieved. The vanishing factor $\gamma_{i}=\frac{1}{i}$, it must be chosen as $\sum_{i} \gamma_{i}=+\infty[1]$.

So that

$$
\mathbf{C}_{i}=\frac{1}{k+1}\left(\sum_{i=0}^{k} \mathbf{p}_{i} \mathbf{p}_{i}^{T}+(k+1) \overline{\mathbf{p}}_{k} \overline{\mathbf{p}}_{k}^{T}\right)
$$

where $\overline{\mathbf{p}}_{i}=\frac{1}{i+1} \sum_{k=0}^{i} \mathbf{p}_{k}$ as in [6]. The covariance matrix is updated as in [8]

$$
\begin{aligned}
\mathbf{C}_{i+1} & =\left(1-\gamma_{i+1}\right) \mathbf{C}_{i}+\gamma_{i+1}\left(\mathbf{q}_{i}-\overline{\mathbf{p}}_{i}\right) \\
\overline{\mathbf{p}}_{i+1} & =\left(1-\gamma_{i+1}\right) \overline{\mathbf{p}}_{i}+\gamma_{i+1} \mathbf{q}_{i}
\end{aligned}
$$

The empirical covariance matrix and the mean proposal are updated as follows The target $x_{o b j}=0.234$ is chosen thanks to [4]. Since the a posteriori distribution can be really degenerate the use of $\mathbf{C}$ and the global factor adaptation allows to sample well the distribution, even if highly degenerated.

\section{Test-cases, numerical application}

The real parameter will be

$$
\mathbf{p}^{*}=\left(\begin{array}{c}
\varepsilon_{C} \\
k \\
S
\end{array}\right)=\left(\begin{array}{c}
0.2510^{-3} \\
0.5 \text { or } 0.75 \text { or } 0.95 \\
70 \mathrm{~ms}^{-1}
\end{array}\right)
$$

In the following figures the cross represents $\mathbf{p}^{*}$, the observations $\hat{\mathbf{M}}$ are the values of the simple moments of $\mathbf{p}^{*}$.

We use the following bounds for the a priori distribution

$$
\begin{aligned}
\varepsilon_{C, \text { min }} & =0.110^{-2} \\
\varepsilon_{C, \text { max }} & =0.410^{-2} \\
k_{\text {min }} & =0 \\
k_{\text {max }} & =1 \\
S_{\text {min }} & =20 \\
S_{\text {max }} & =200
\end{aligned}
$$


The quantity of interest is $N_{[0, T]}$ when $T=10 \mathrm{~ms}$ and for a time of measurement of $T_{\text {meas }}=$ 36, 360, $3600 \mathrm{~s}$. We have also considered $\alpha_{Y}=2 \mathrm{~ms}^{-1}$ and

$$
\begin{aligned}
\bar{\nu} & =2.4130 & \bar{\nu}_{S} & =1.000 \\
D_{2} & =0.7992 & D_{2 S} & =0 \\
D_{3} & =0.4819 & D_{3 S} & =0
\end{aligned}
$$

The initialization parameters of the AM algorithm are

$$
\begin{aligned}
x_{\text {rate }} & =1 \\
x_{o b j} & =0.234 \\
f r a c & =0.1 \\
N_{b p} & =10^{7} \\
N_{M C, 1} & =\max \left(\frac{N_{M C}}{10000}, 1\right)
\end{aligned}
$$

Regarding the explicit sampling there are $N_{e}^{3}$ points in the grid with $N_{e}=400$.

The $2 \mathrm{D}$-a posteriori distribution of the parameter $\mathbf{p}$ are estimated by histograms with $100 \times 100$ from the AM sample.
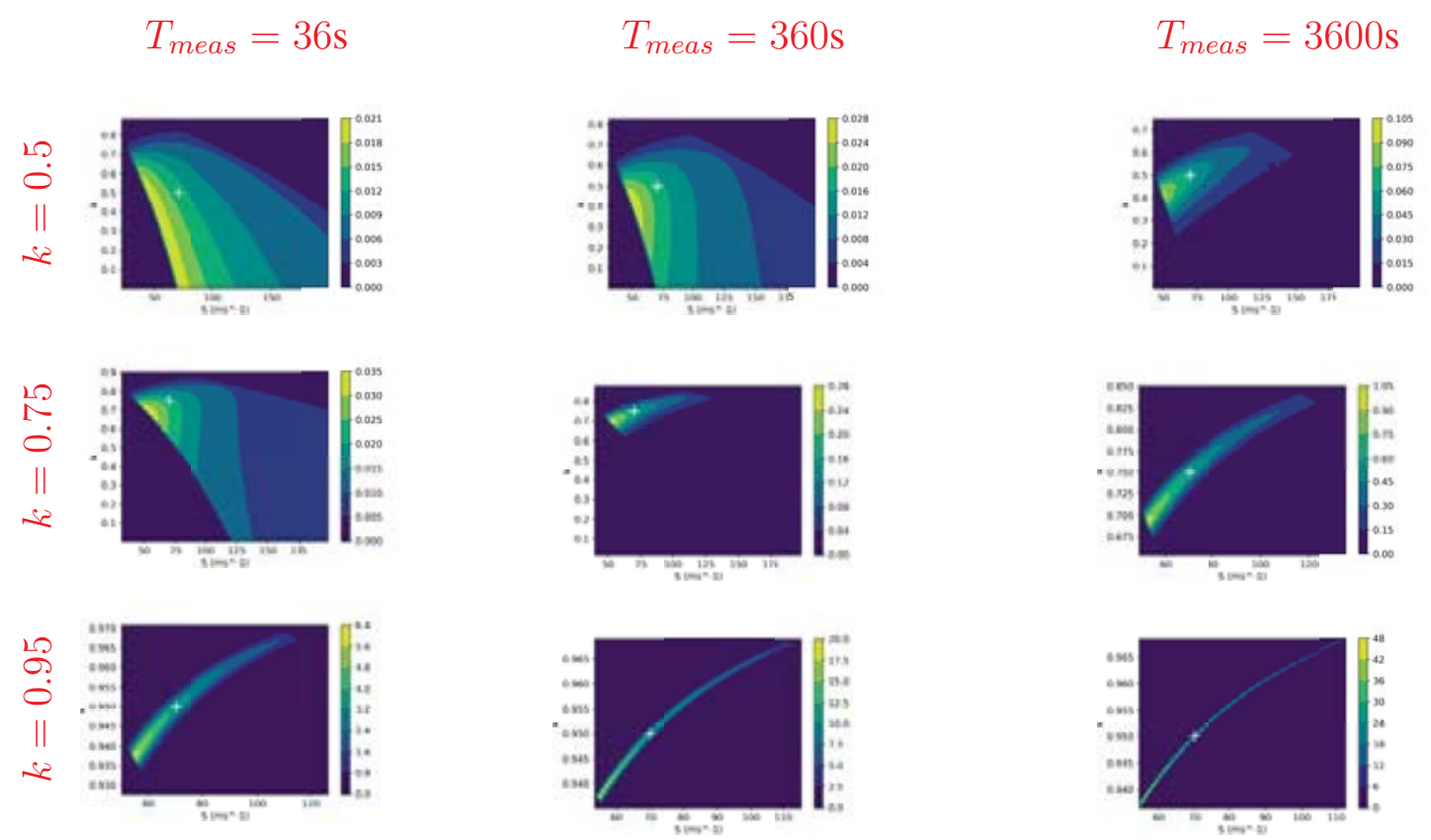

Figure 2: A posteriori distribution for $(k, S)$ using 3P3M with explicit sampling

We can compare it to the result using the MCMC method 

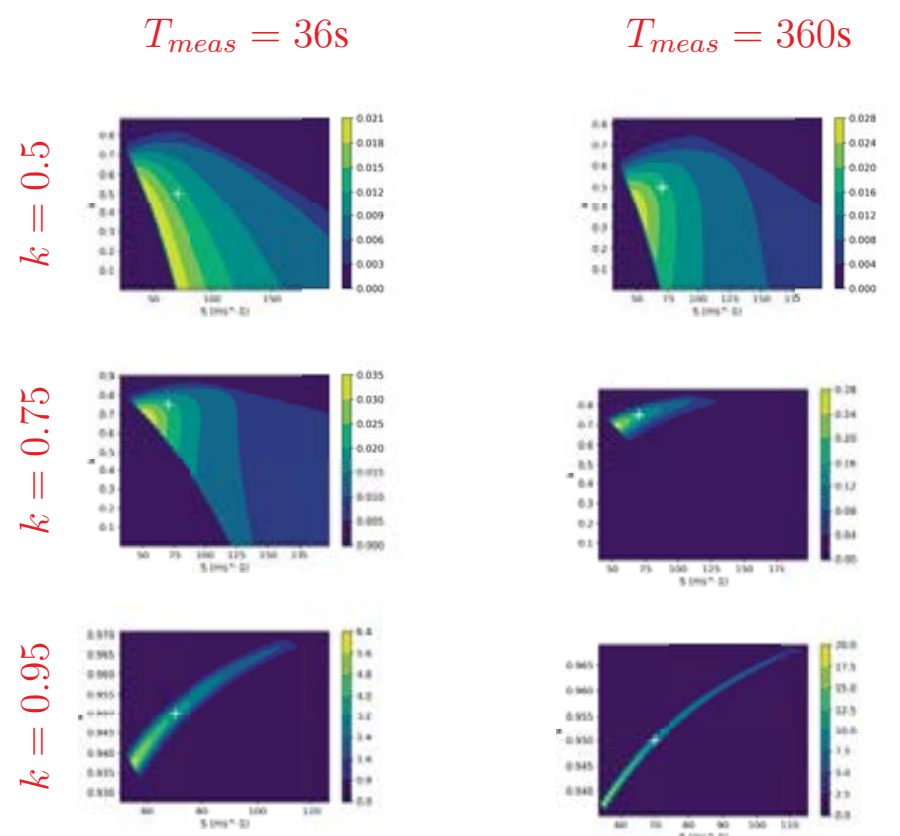

$$
T_{\text {meas }}=3600 \mathrm{~s}
$$
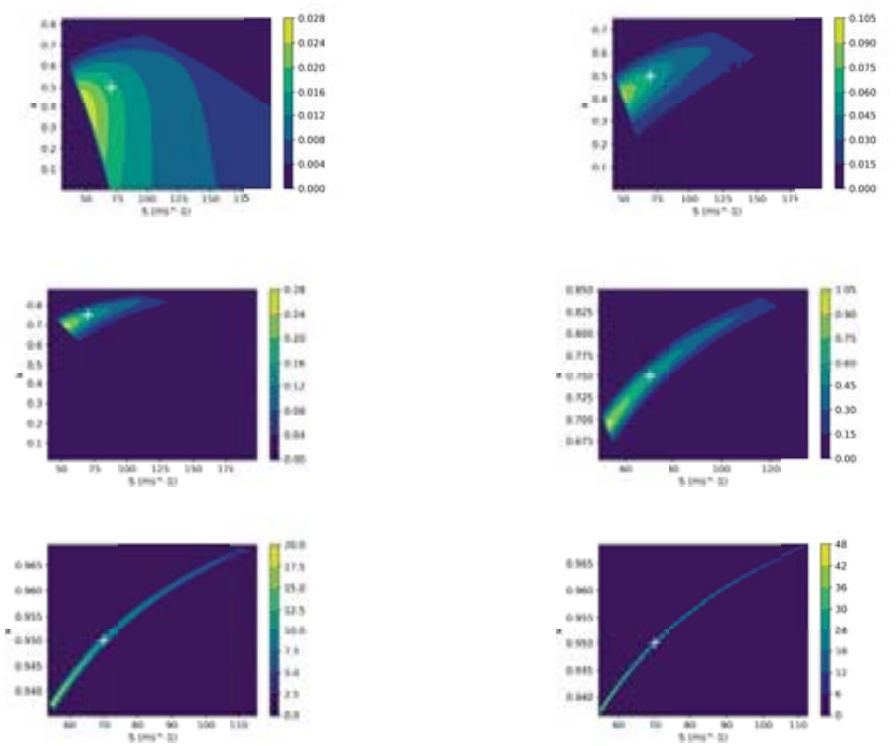

Figure 3: A posteriori distribution for $(k, S)$ using 3P3M with MCMC sampling

We can also observe the a posteriori distribution for $\left(k, \varepsilon_{C}\right)$
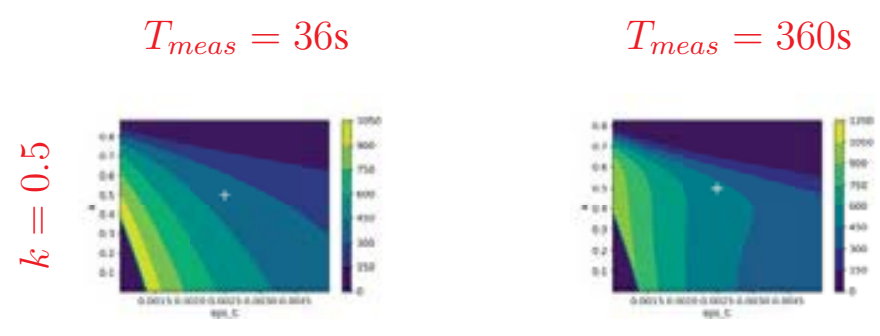

$$
T_{\text {meas }}=3600 \mathrm{~s}
$$
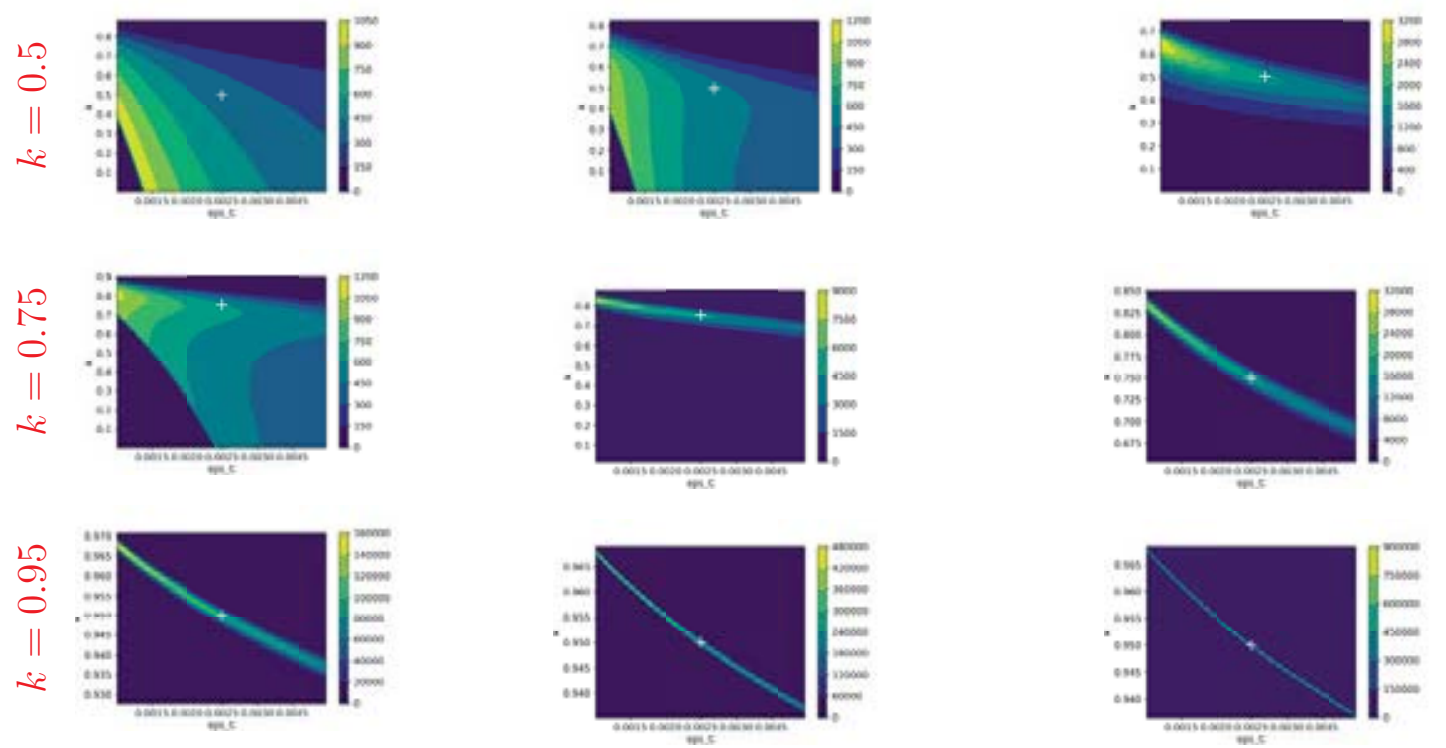

Figure 4: A posteriori distribution for $\left(k, \varepsilon_{C}\right)$ using 3P3M with explicit sampling

We can compare these results to the MCMC method results 


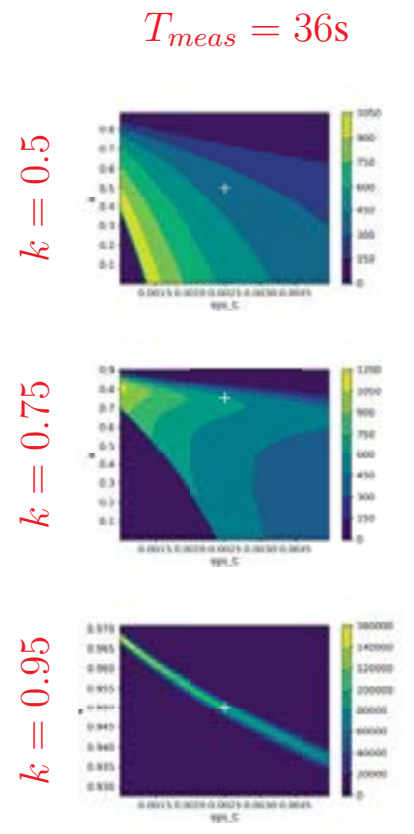

$T_{\text {meas }}=360 \mathrm{~s}$
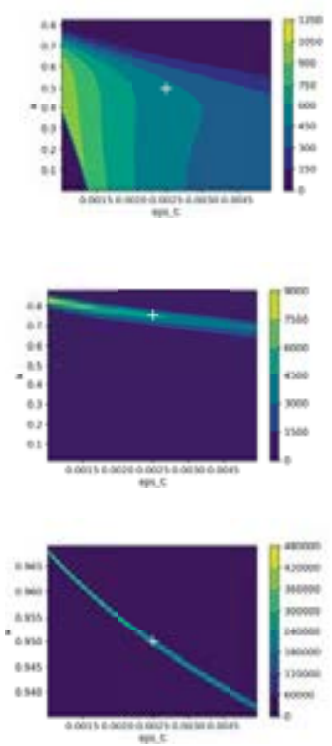

$T_{\text {meas }}=3600 \mathrm{~s}$
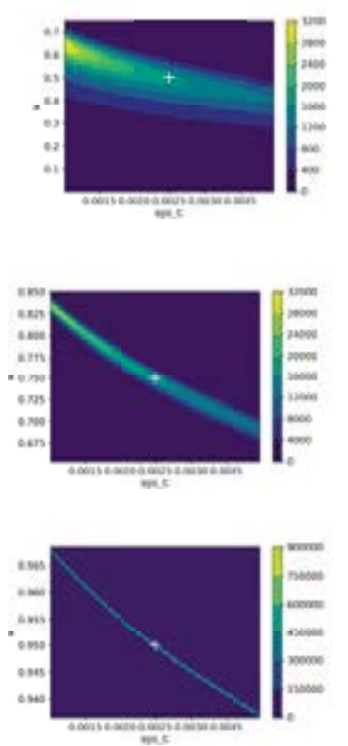

Figure 5: A posteriori distribution for $\left(k, \varepsilon_{C}\right)$ using 3P3M with MCMC

\section{Discussion}

The explicit sampling of the distribution is really effective when the dimension of $\mathbf{p}$ is up to three.

When the dimension is larger than four, the AM algorithm is efficient whatever the dimension of $\mathbf{p}$ is.

We observe the higher the multiplication factor $k$ the more the distribution is degenerate. The same effects appear when the time of measurement $T_{\text {meas }}$ is large. The real value $\mathbf{p}^{*}$ is in the support of the distribution, but this support is large.

\section{Conclusion}

To sum up, in the context of the neutron point model we have used the analytic expression of the three first simple moments which define the forward model of our inverse problem. Our observations are the estimation of the three first empirical moments of the neutron count distribution. Then using the Bayes principle, we have exposed the estimation of the a posteriori distribution of the parameters. Then we have implemented two sampling methods of this a posteriori distribution:

- The first method is a simple sampling with a regular grid whose cost dramatically increases with the dimension of the parameter.

- The second method is obtained by the use of the Adaptive Metropolis algorithm with Covariance Matrix Adaptation

On an example with synthetic data, we observed that the support of the distribution contains the true parameter. The distribution is more degenerate when the multiplication factor $k$ is high, and also when $T_{\text {meas }}$ is large.

The explicit sampling is well adapted when $\operatorname{dim}\left(\mathbf{p}^{*}\right) \leq 3$, but it is too expensive when this condition is not satisfied. Then the use of the AM-CMA approach is required. 
The present work shows that the sampling (explicit or with AM-CMA) is satisfactory to retrieve the true for parameter for one time gate $T$ when $\operatorname{dim}\left(\mathbf{p}^{*}\right) \leq 3$. Considering two time gates $T_{1}$ and $T_{2}$ will enable recovering a parameter of higher dimension.

\section{REFERENCES}

[1] C. Andrieu, J. Thoms A tutorial on adaptive MCMC, Stat Comput, 18, 343-373, 2008

[2] G. I. Bell and S. Glasstone, Nuclear Reactor Theory, 1970, Van Nostrand Reinhold Company

[3] R. P. Feynman, F. de Hoffmann, R. Serbe, Statistical fluctuations in the water boiler and the dispertion of neutrons emitted per fission, 322, 10, 891-921, 1944

[4] A. Gelman, G.O. Roberts, W. R. Gilks, Efficient Metropolis Jumping Rules, Bayesian Statistics, 5, 599-607, 1996

[5] H. Haario, E. Saksman and J Tamminen, Adaptive proposal distribution for random walk Metropolis algorithm, Computational Statistics, 14, 1, 375-395, 1999

[6] H. Haario, E. Saksman and J Tamminen, An adaptive Metropolis algorithm, Bernoulli, 7, 2, 223-242, 2001

[7] P. Humbert, Simulation and Analysis of List Mode Measurements on SILENE Reactor, Journal of Computational and Theoretical Transport, 47, 4-6, 350-363, 2018

[8] C. L. Müller, Exploring the common concepts of adaptive MCMC and Covariance Matrix Adaptation schemes Dagstuhl Seminar Proceedings 10361, Theory of Evolutionary Algorithms

[9] I. Pazsit, L. Pal, Neutron Fluctuations A Treatise on the Physics of Branching Processes, 2008, Elsevier Ltd, London, New York, Tokyo

[10] C. P. Robert and G. Casella. Monte Carlo Statistical Methods. 2004, Springer Texts in Statistics. Springer-Verlag, New York

[11] J. Saxby, Numerical Solution of the Phase-Space Dependent Backward Master Equation for the Probability Distribution of Neutron Number in a Subcritical Multiplying Sample, Imperial College London Department of Mechanical Engineering, 2017

[12] T.J. Sullivan, Introduction to Uncertainty Quantification, 2015, Springer International Publishing

[13] A. Tarantola, Inverse Problem Theory and Methods for Model Parameter Estimation, 2005, SIAM

[14] J. Terrell, Distribution of Fission Neutron Numbers, Physical Review, 108, 3, 783-789, 1957

[15] N. Tsoulfanidis, S. Landberger, Measurements \& Detection of radiation $4^{\text {th }}$ edition, 2015, CRC Press 